\title{
POPULAÇÃO EM SITUAÇÃO DE RUA, VULNERABILIDADES E DROGAS: UMA REVISÃO SISTEMÁTICA
}

\author{
POBLACIÓN SIN HOGAR, VULNERABILIDADES Y DROGAS: \\ UNA REVISIÓN SISTEMÁTICA
HOMELESS POPULATION, VULNERABILITIES AND DRUGS: A STSTEMATIC REVIEW

\author{
Kíssila Teixeira Mendes ${ }^{1}$, Telmo Mota Ronzani ${ }^{1}$ \\ e Fernando Santana de Paiva ${ }^{1}$
}

${ }^{1}$ Universidade Federal de Juiz de Fora, Juiz de Fora/MG, Brasil

\begin{abstract}
RESUMO: O presente artigo tem como objetivo realizar uma revisão sistemática sobre os determinantes pobreza, estigma, raça e vulnerabilidade entre a população em situação de rua usuária de drogas. Para tal, foi realizada uma revisão sistemática utilizando as bases de dados Scielo Br, PepSiC, PubMed e Redalyc. Ao final dos procedimentos foram selecionados 79 artigos. Foi percebida diferença significativa dos estudos realizados nos Estados Unidos e os latinoamericanos. Enquanto os primeiros tendem a priorizar estudos com grandes amostras com o foco na análise de riscos e na quantificação de categorias, as pesquisas latinas tendem a contextualizar as condições de desigualdade e pobreza, ao passo que utilizam metodologias participativas e se aproximam de uma análise das vulnerabilidades. Os resultados indicam discussões ainda incipientes que articulem tais categorias e que pouco englobam as diversas vulnerabilidades a que esses sujeitos estão expostos ao longo de suas vidas, bem como apontam para a necessidade de uma nova categoria analítica de compreensão.
\end{abstract}

PALAVRAS CHAVE: População em situação de rua; Drogas; Vulnerabilidade; Pobreza; Estigma.

RESUMEN: El presente artículo tiene como objetivo realizar una revisión sistemática sobre los siguientes determinantes: pobreza, estigma, raza y vulnerabilidad entre la población sin hogar usuaria de drogas. Para ello, se realizó una revisión sistemática utilizando las bases de datos Scielo Br, PepSiC, PubMed y Redalyc. Al final de los procedimientos se seleccionaron 79 artículos. Se percibió una diferencia significativa de los estudios realizados en los Estados Unidos y los latinoamericanos. Mientras que los primeros tienden a priorizar estudios con grandes muestras con el foco en el análisis de riesgos y en la cuantificación de categorías, las investigaciones latinas tienden a contextualizar las condiciones de desigualdad y pobreza, utilizando metodologías participativas y se aproximando a un análisis de las vulnerabilidades. Los resultados indican discusiones aún incipientes que articulan tales categorías y que poco engloban las diversas vulnerabilidades a que estos sujetos están expuestos a lo largo de sus vidas, así como apuntan a la necesidad de una nueva categoría analítica de comprensión.

PALABRAS CLAVE: Población sin hogar; Drogas; Vulnerabilidad; Pobreza; Estigma.

ABSTRACT: This article aims to make a systematic review of the following determinants: poverty, stigma, race and

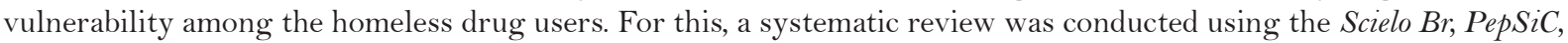
PubMed and Redalyc databases. At the end of the procedures 79 articles were selected. Significant difference was noted in the studies conducted in the United States and in Latin America. While the former tends to prioritize studies with large samples focused on risk analysis and quantification of categories, Latin researches tend to contextualize the conditions of inequality and poverty, while using participatory methodologies and approach an analysis of vulnerabilities. The results indicate incipient discussions that articulate these categories and include some the various vulnerabilities to which these individuals are exposed throughout their lives, as well ååas point to the need for a new analytical category of understanding regarding this matter.

KEYWORDS: Homeless; Drugs; Vulnerability; Poverty; Stigma. 


\section{Introdução}

Com o objetivo de realizar uma revisão sistemática sobre os determinantes pobreza, estigma, raça e vulnerabilidade entre a população em situação de rua usuária de drogas, o presente artigo procura refletir, a partir do referencial teórico de Psicologia Comunitária e Saúde Coletiva, sobre a utilização das categorias "risco" e "vulnerabilidade" no debate sobre saúde e condições de vida da população.

Souza, Silva e Silva (2013), relatam que o movimento do campo da saúde em abarcar fatores econômicos, sociais e ambientais (enfoque social), se afastando de concepções puramente biológicas (enfoque biológico), se inicia recentemente, no século XX, com a denominação dos determinantes sociais da saúde (DSS). Essa mudança de paradigma, de fato, foi fundamental para uma melhor compreensão sobre saúde e doença, a partir do momento em que englobam as condições de vida e pobreza, raciais, de gênero e de trabalho, entre outras.

No entanto, a partir do recorte sobre a população em situação de rua - os mais pobres entre os pobres, o debate nos exigiu também compreender como as análises sobre pobreza - e a própria desigualdade, em si - impactam na vida da população. Façamos antes, porém, uma breve leitura sobre as principais categorias da revisão sistemática - população em situação de rua, vulnerabilidade e droga.

A população em situação de rua aparece como um segmento expressivo da desigualdade social, sobretudo a partir das mudanças políticas, socais e econômicas mundiais das últimas décadas. Apresenta-se como uma categoria social complexa, fundamentalmente por conta da necessidade de se considerar o processo de "vulnerabilização" como dinâmico e anterior à ida para as ruas. Existe uma tendência, proveniente de abordagens contemporâneas, em se analisar e compreender as particularidades em que vivem essa população, principalmente no âmbito da saúde pública, a partir de uma redução desta condição complexa a um enfoque individualista e patologizante.

Além disso, o uso e abuso de drogas, sintomas ou quadros de adoecimento psíquico são empregados como fatores de causalidade para a condição de se viver nas ruas. É importante salientar que, apesar das particularidades das experiências vivenciadas, não é possível individualizar a condição da rua, pois esta, como antes citado, tem intrínseca relação com fatores macroestruturais de ordem socioeconômica e política, em especial, quando analisamos a formação histórica brasileira, marcada por um abismo social entre os diferentes segmentos de classe social. Logo, a análise sobre as trajetórias dos sujeitos em condição de vulnerabilidade podem contribuir para ampliar nossa compreensão sobre a PSR (Varanda \& Adorno, 2004). No entanto, do que se trata a categoria vulnerabilidade? Partindo da leitura dos DSS, a adoção do termo vulnerabilidade enquanto conceito ampliado é mais viável, pois, teoricamente, lida com a limitação ao acesso de direitos e bens imposta pela desigualdade. Debateremos, ao longo do artigo, as contradições desse termo a partir da revisão sistemática e apontaremos para outras possibilidades de análise, sendo este o caráter original do trabalho.

O uso de drogas, por sua vez, é intimamente ligado à história da humanidade. Ao uso são atribuídos diferentes significados e valores e, por isso, se faz necessário compreender sua complexidade e a multifatoriedade (Ronzani \& Furtado, 2010). Entretanto, atualmente, o uso abusivo de drogas está articulado aos quadros de desigualdades sociais, sendo objeto de diversas políticas públicas (Souza \& Kantorski, 2007). Porém, é também de alguma forma consensual, que nem todo uso de drogas é problemático (Mayora, 2016). Apesar 
disso, o uso de drogas carrega um forte aspecto moralizante que, reduzido a uma questão individual, culpabiliza o usuário por sua condição a partir de um viés estigmatizante (Ronzani \& Furtado, 2010). O estigma se constitui, assim, como um elemento importante na equação das opressões e dominações e se origina de processos históricos e políticos de constituição de regimes políticos normativos (Parker \& Aggleton, 2001).

Por isso, Rui (2006) tensiona que o uso de drogas só pode ser compreendido a partir da ressalva do marcador de classe. $\mathrm{O}$ uso de drogas atribui barreiras dentro da sociedade, havendo legitimações de quem pode e tem controle para fazer ou não o uso da droga. Assim, é fundamental saber quem consome, o local e a forma com que o faz. Fica claro, dessa forma, que há a necessidade de ver o uso de drogas como fenômeno coletivo, e não individual (Alvarado, Guerra, \& Mejías, 2013). Mayora (2016) alerta, então, para a necessidade de a droga, por si própria, não ser o ponto principal de análise, sendo ela um desafio entre tantos outros encontrados na rua. Assim, em um contexto de extremo pauperismo, como na rua, a análise das implicações do uso de drogas parece mais complexo: "o que seria um consumo problemático ou não problemático na vida de quem não tem nada, de quem passa fome, frio e dorme no chão?” (Mayora, 2016, p. 159).

Por isso, entre as categorias pesquisadas no presente artigo (drogas, população em situação de rua, vulnerabilidade, pobreza e raça), a pobreza aparece como conceito fundamental, como ficará evidente. Entretanto, um projeto emancipatório se dá, além do terreno econômico, também a partir de batalhas no terreno extra econômico. Por isso, é necessário o diálogo entre os demais marcadores sociais - tais como a raça, que são importantes ao expressarem relações de poder e dominação. Assim, os diversos tipos de opressão não são autônomos e devem ser vistos como parte integrante da sociedade capitalista (Arruza, 2015). Tais marcadores, mais uma vez, dizem de trajetórias de marginalização social e vulnerabilidades que antecedem o uso da droga e a ida para as ruas. No entanto, como a literatura tem, historicamente, os abordado? A partir da revisão sistemática da literatura, buscaremos responder essa questão e refletir sobre seus tensionamentos.

\section{Métodos}

O presente estudo é uma revisão sistemática, que segundo Galvão, Sawada e Trevizan (2004, p. 1), trata-se de "um recurso importante da prática baseada em evidências, que consiste em uma forma de síntese dos resultados de pesquisas relacionados com um problema específico". Sendo assim, busca verificar, com alta força de evidência científica e com métodos bem delineados, o desenvolvimento ou o "estado da arte" de determinadas temáticas. Para tal, foram utilizados quatro bancos de dados eletrônicos, a saber: Scielo Br; PepSiC; PubMed e Redalyc. A escolha das bases de dados se deve à relação do tema com o conteúdo indexado, bem como a variedade de países pesquisados e áreas de saber que as bases apresentam. Foram realizadas pesquisas com os termos nas línguas portuguesa, inglesa e espanhola.

Os termos pesquisados foram definidos de acordo com o objetivo do trabalho e são: drogas, população em situação de rua, pobreza, estigma, raça e vulnerabilidade. Os resultados de cada um dos termos foram cruzados com o termo "drogas" utilizando o operador booleano " $A N D$ " com a finalidade de restringir a pesquisa aos resumos que apresentavam ao mesmo tempo os dois termos. Entretanto, devido a pouca precisão dos resultados 
encontrados, houve diferentes estratégias de busca nos diferentes bancos. No PubMed, devido ao número muito expressivo de resultados utilizando apenas dois termos cruzados, foi estabelecido como padrão os termos "drugs AND homeless". No caso do Scielo Br e do PepSiC, foi mantida a pesquisa com dois termos, com o termo "droga" como padrão. No Scielo $B r$, ainda, devido a pouca quantidade de resultados, ao invés de "população em situação de rua" foi utilizado apenas o termo "rua" durante as pesquisas em português e espanhol com a finalidade de ampliar as possibilidades. No banco de dados Redalyc não há a opção de pesquisa com mais de três descritores. Por isso, o padrão utilizado foi, ora o termo "drogas" ora o termo "população em situação de rua", utilizando o operador booleano "Y” em ambas as línguas.

Os critérios de inclusão utilizados foram: (a) artigos originais e completos nas línguas inglesa, espanhola e portuguesa. (b) pesquisas empíricas, apresentadas em formato de artigo, com foco na população de rua, com base em fonte primária. Considerou-se pesquisas com diferentes abordagens de pesquisa, a exemplo, estudos etnográficos e estudos de caso, de natureza quantitativa e qualitativa. Foram excluídos artigos teóricos e/ou baseados em relatórios estatísticos obtidos de fontes secundárias, como os estudos que visavam a análise de instituições que acolhem pessoas em situação de rua. A partir de tais critérios foram selecionados 79 artigos (22 no PubMed, 10 no Scielo, 2 no PePSiC e 45 no Redalyc) na fase quantitativa da análise. Posteriormente, foi realizada a leitura dos resumos e a leitura flutuante dos artigos, bem como foram excluídos os artigos duplicados. Estes foram organizados a partir de algumas informações, tais como: número de informantes da pesquisa, autores, revista, método/instrumentos, área de atuação dos autores, ano, local e país. Nos delineamentos quantitativos a seguir, serão apresentados dados dos 79 artigos selecionados.

Após esse procedimento, foi realizada uma leitura com foco na análise das categorias raça, pobreza, estigma e vulnerabilidade. Os artigos foram organizados da seguinte forma: ausência de categorias (nenhuma delas é citada no trabalho); é citada sem uma discussão aprofundada; ao menos uma das categorias, há teorização ou debate (trabalhado). Assim, ao final, 40 artigos foram considerados com debates relevantes à revisão (qualitativa) e considerados na discussão.

\section{Resultados}

Os anos de publicação dos 79 artigos que compõem a revisão variam entre 1990 a 2015. O período de 1990 a 1995 teve 4 publicações; de 1996 a 2000, 6 publicações; de 2001 a 2005 foram 8 publicações; e de 2006 a 2015 um avanço no número de publicações, com 61 . O início dos estudos realizados nos Estados Unidos datam de 1990, seguindo um fluxo contínuo de publicações 2015. Os trabalhos realizados na América Latina, por sua vez, têm a primeira publicação datada de 1997, sendo que não há uma linearidade de publicações. Após os anos 2000, fundamentalmente de 2006 a 2015, há um maior interesse na temática pelos pesquisadores latino-americanos, considerando o maior acúmulo dos estudos nesse período.

Os países em que foram realizadas as pesquisas foram diversos, sendo o Brasil o com maior número de publicações, totalizando $43 \%^{1}$ das pesquisas. É seguido pelos Estados Unidos (22,8\%), Colômbia (10\%), México (5\%). Venezuela (3,8\%), Canadá (2,5\%), Chile $(2,5 \%)$, Cuba (1,3\%), Equador (1,3\%), Argentina (1,3\%), França (1,3\%), Gana (1,3\%), 
Inglaterra (1,3\%), Peru (1,3\%), e uma pesquisa sem indicação de local (1,3\%). As áreas de saber também foram variadas, sendo a com maior frequência a Psicologia (24\%) seguida por: Saúde Coletiva/ Comunitária/ Epidemologia (22,2\%); estudos multidisciplinares, com duas ou mais áreas diferentes (17,7\%); Medicina (12,7\%); Enfermagem (9\%); Ciências Sociais (6,5\%); Saúde, sem especificação (5\%); Assistência Social (1,3\%); e Terapia Ocupacional (1,3\%).

Dos 79 artigos, 58,8\% apresentaram como enfoque principal a relação das pessoas em situação de rua com as drogas. Entre esses, em 65,2\% o tipo de droga não era especificado, considerando drogas de forma geral. $\mathrm{O}$ crack aparece como a droga específica mais estudada (15,2\%), seguida pelo álcool (8,7\%), por medicações de prescrição médica (4,3\%) e por tabaco, heroína e metanfetamina, ambos com $2,2 \%$ cada.

Quanto à população estudada, 73,41\% das pesquisas apresentaram um foco em uma determinada população. Nota-se enfoque majoritário na pesquisa com crianças e adolescentes em situação de rua, com $45,56 \%$ do total destinadas a esse público. Em seguida, o estudo com mulheres em situação de rua, com 11,39\%; com população LGBTTT (Lésbicas, Gays, Bissexuais, Travestis, Transexuais e Transgêneros), com 6,32\%; com famílias sem teto, com 5\%; com negros, com 2,53\%; e com idosos e homens, ambos com 1,25\% cada. O estudo de condições de saúde específicas foi frequente, com um total de 21,5\%, sendo $58,8 \%$ deles localizados na base de dados PubMed (que possui a característica de estudos voltados para a área da biomedicina e ciências da vida). Das pesquisas que estudam condições de saúde, $64,7 \%$ das pesquisas são sobre $H I V / A I D S$, 17,6\% sobre Hepatites, 5,9\% sobre gravidez, $5,9 \%$ sobre sífilis e 5,9\% sobre transtornos psiquiátricos.

A análise quantitativa prévia dos estudos indica, dessa forma, uma série de debates possíveis. Há diferenças significativas entre os estudos estadunidenses e os latino-ameri$\operatorname{canos}^{2}$, sendo essa, então, a linha de raciocínio escolhida para a discussão, a seguir será apresentada uma análise dos resultados encontrados, delimitando os estudos realizados no cenário estadunidense e latino-americano.

\section{Discussão}

\section{Os trabalhos estadunidenses ${ }^{3}$ e do norte: o conceito de "risco" e as vulnerabilidades transformadas em categorias mensuráveis}

Os trabalhos norte-americanos, fundamentalmente, partem de uma concepção pautada pelo conceito de risco, usual no campo da saúde até meados dos anos 1990 a partir de uma perspectiva individualizante (Ayres, França, Calazans, \& Saletti, 2009). Assim, se utilizam de uma análise calcada em condições físicas ou biológicas e com a associação de doenças a públicos específicos. Nesses estudos, as vulnerabilidades transformam-se em categorias quantitativas e mensuráveis. A categoria raça, por exemplo, é citada diversas vezes como dado sociodemográfico (Bassuk et al., 1997; Hennessey et al., 2009; Kecojevic et al., 2012; Lankenau et al., 2010; Nyamathi et al., 2002; Riley et al., 2011; Vogenthaler et al., 2010; Wechsberg et al., 2004; Weiser et al., 2006; Whittle et al., 2015; Wood et al., 1990), bem como pobreza é tida meramente como indicador de renda, sendo usual termos como "linha da pobreza" (Bassuk et al. 1997; Lewis et al., 1995; Vogenthaler et al., 2010), 
"nível de pobreza" (Wechsberg et al., 2004; Wood et al., 1990 ) e "contexto social da pobreza" (Riley et el., 2011), sem definições claras e/ou um debate estrutural sobre raça e pobreza. A categoria vulnerabilidade, por sua vez, é a mais heterogênea, carregando inúmeros sentidos, sem nenhuma definição teórica mais enfática, o que é reflexo da própria dificuldade de definição da categoria (Janczura, 2012). Se caracterizam também por serem estudo com grandes amostragens, sendo o menor com 34 participantes (Whittle et al., 2015) e o maior com 1985 participantes (Bobashev et al., 2009).

Os estudos encontrados na revisão apontam para metodologias comparativas (Gattis, 2013; Wenzel et al., 2012) e para a inter-relação de variáveis (Bobashev et al., 2009; Shoptaw et al., 2009), sendo analisadas as categorias: sexualidade, raça, gênero, pobreza e estigma. Além disso, partem de uma leitura a partir do conceito de "risco". Se, por um lado, esses estudos adicionam condições de vulnerabilidade, como a sexualidade, na trajetória do sujeito, afastando a ideia de homogeneidade da população em situação de rua, e alertam para maior frequência e risco de condições de saúde como Hepatite A (Hennessey et al., 2009) e HIV (Shoptaw et al., 2009) em pessoas em situação de rua e para a necessidade de programas preventivos específicos voltados a essa população; por outro, carecem de um debate aprofundado acerca de tais categorias, que aparecem como naturalizadas, sem que sejam abarcadas as raízes do problema. O grande problema desta determinação objetal da doença, segundo Nogueira (2010), é o discurso de neutralidade atrelado a ele. Quando pensamos na área da saúde, ainda "é preciso sempre ter em vista que a determinação objetal da doença está subordinada ao propósito de estabelecer um domínio das ciências médicas sobre a pessoa humana em sua totalidade" (Nogueira, 2010, p. 149).

Verifica-se, assim, a partir de um compilado dos estudos coletados, a preocupação, plausível, com a associação entre comportamentos específicos da população em situação da rua e suas consequências, como as doenças sexualmente transmissíveis, a discriminação e o uso de drogas. Entretanto, se analisarmos a partir de uma concepção mais ampla, não deveria de tratar de compreender somente a susceptibilidade a determinadas doenças ou condições, e sim de abarcar contextos e trajetórias que culminaram na situação de rua (e o termo situação é apropriado por, justamente, indicar uma condição não estática) e sua intersecção com as particularidades dos sujeitos (Alvarado, Guerra, \& Mejías, 2013).

Sánchez e Bertolozzi (2007), em revisão de estudos da área de saúde, buscam uma compreensão de como o termo "vulnerabilidade" vem sendo utilizado nessa área. Concluem uma multiplicidade de possibilidades, que passam desde simples a capacidade de enfrentamento a adversidades, sendo estritamente individual, até definições multifatoriais, que incluem noções relativas ao direito das pessoas, à economia e à participação política e institucional. Essa última definição compreende que a vulnerabilidade se manifesta de forma diferente nos sujeitos em relação às regiões, momento histórico e a história de vida individual. Dessa forma, busca a superação do modelo biológico tradicional e adota como referencial de intervenção a comunidade, acreditando que somente em conjunto com a população é possível encontrar alternativas de mudança.

Esses modelos buscam uma superação do conceito de "risco", usual na Epidemiologia, incorporando questões estruturais e do plano singular. No campo da saúde tem-se usado historicamente o conceito de risco, como é perceptível nos trabalhos norte americanos, como elemento probabilístico que orienta as ações da área de maneira individualizante. Sánchez e Bertolozzi (2007) propõem, então, o uso do conceito de vulnerabilidade como "potencial instrumento para a transformação das práticas de saúde" (Sánchez \& 
Bertolozzi, 2007, p. 4). Assim, os contornos da questão social são vistos com diferentes enfoques a partir do contexto sociopolítico (Kowarick, 2003). Veremos adiante, então, como as mesmas questões são compreendidas a partir da realidade latino-americana e debater as categorias centrais do artigo a partir desse ponto.

\section{Os trabalhos latino-americanos: a inclusão de determinantes econômicos, políticos e sociais}

As pesquisas latino-americanas, em grande medida, partem da necessidade de uma análise que englobe os DSS e discussões de âmbito político, social e econômico. Sendo assim, um primeiro ponto importante de análise destes estudos e que já os diferencia dos estudos antes citados, é o método predominante: destes, 14 estudos se declaram como etnográficos, sendo usuais os estudos de caso, as entrevistas em profundidade, as histórias de vida, a pesquisa ação e a pesquisa participante. Adorno et al. (2013) apontam para a necessidade de mudanças teóricas e metodológicas no estudo de populações em situação de rua, em específico, e indica a etnografia como perspectiva metodológica capaz de articular questões macroestruturais e temáticas urbanas e deixar mais claras as margens do próprio Estado. As metodologias mais participativas, segundo os autores, têm maior capacidade de ampliação do debate, bem como de desconstrução do pensamento, visto que articula dinâmicas sociais e aspectos das trajetórias de vida.

Quanto às categorias de análise pesquisadas para a revisão, o uso de drogas, aparece tanto como causa como consequência das ruas, sendo associado à vulnerabilidade familiar - aqui compreendida a partir do critério de renda, à falta de afeto e a maus tratos físicos e, após a chegada nas ruas, como possibilidade de maior interação social e como forma de cumprir as exigências de pertencimento e sociabilidadade dos centros urbanos (Alvarado, Guerra, \& Mejías, 2013; Moro, Silva, \& Noto, 2009; Giraldo et al., 2008). Já a categoria pobreza vem como característica em comum entre usuários de drogas pesquisados nos cenários de uso (Ferreira et al., 2015) e também como principal causa de ida para às ruas (Martins, 2002). O único trabalho que refuta a ideia de que a pobreza é causa direta de ida de crianças à rua é de Mizen e Kusi (2014).

É nítida, como apontado nos resultados, a prevalência de estudos de crianças e jovens. Há apenas um estudo de caso sobre idoso em situação de rua (Brêtas et al., 2010). Os adolescentes são colocados ainda como grupo de maior vulnerabilidade no contexto da rua, fundamentalmente em relação ao uso de drogas e quando associada a categoria gênero (Chacón et al, 2011; Nunes \& Andrade, 2009). Rosa e Brêtas (2015) atenta também para a não existência de políticas específicas para mulheres nessa condição. Além disso, o uso de drogas é visto como mais estigmatizante quando vinculado à mulher (Chacón et al., 2011). A relação com o corpo e a vulnerabilidade a doenças sexualmente transmissíveis também são foco de análise. Grangeiro et al., (2012) retratam a prevalência e o perfil de vulnerabilidade ao HIV entre moradores de rua na cidade e São Paulo e caracterizam como grupo de maior vulnerabilidade as mulheres, os jovens e os homossexuais. Esses fatores levam a situações de maior exposição ao HIV, como a violência e a falta de acesso aos serviços.

É possível observar ainda uma ênfase da instituição família nos artigos encontrados, influenciados pela lógica (perversa), apoiada no ideário neoliberal, de que, assegurado o sustento mínimo, a família e, sobretudo, a mulher, pode ser responsável pelas funções de bem estar de seus indivíduos. Sunkel (2006), então, indica a necessidade de superação do foco nas famílias conforme modelo tradicional. 
A raça é muito pouco trabalhada nos estudos latino-americanos, aparecendo como categoria sociodemográfica e considerada em interseção com outras categorias (Barata et al., 2015; Moura, Ximenes, \& Sarriera, 2013; Vernaglia, Vieira, \& Cruz, 2015; Sarcinelli, 2011). Nenhuma pesquisa traz tal categoria como principal elemento para análise. Laguardia (2004) afirma que, aquém do crescimento do uso da variável raça nos estudos de saúde pública, categorias raciais e étnicas continuam, além de negligenciadas, a serem alvos de controvérsia por conta das visões biologicistas que ainda permeiam a área da saúde. Defende ainda que a raça não é indicador biológico, nem tampouco a cor da pele define escolhas individuais e estilos de vida. A raça é, sim, um ótimo indicador do grau de exposição a fatores sociais, sugerindo posições subalternas na hierarquia social (logo, sendo indissociável à classe social).

Moura, Ximenes e Sarriera (2013) são os que trabalham mais claramente as categorias pobreza e estigma na população em situação de rua. O processo de estigmatização é caracterizado como prática de opressão e constituição de identidades. Concluem, assim, que a situação de rua é permeada de elementos que promovem a humilhação e a vergonha, convertendo essas pessoas unicamente à representação de agressivos e usuários de drogas. Alcântara, Abreu e Farias (2015) também relacionam estigma e uso de drogas, ressaltando que as pessoas em situação de rua estão expostas a representações sociais comuns às pessoas pobres, como as de malandragem e de vitimismo.

A caracterização dos artigos encontrados na revisão sistemática, aqui antes apresentados, sugere que os estudos latino-americanos visam as trajetórias de vulnerabilidades, bem como propõem um debate mais aprofundado dos marcadores pesquisados, sob um enfoque dos DSS. Isso implica em leituras do quadro histórico e conjuntural, bem como em debates e defesas claras sobre o sistema de saúde, a participação popular, a proteção social e ao próprio patamar civilizatório - ou ao "modo de vida", partindo de uma compreensão marxiana (Fleur-Teixeira \& Bronzo, 2010). Além disso, possuem as categorias pobreza e vulnerabilidade como centrais, com resultados mais voltados para a realidade objetiva e com apontamentos de superação via políticas públicas. Sendo assim, correspondem à mudança de paradigma antes mencionada. No entanto, nos cabe perguntar o que, de fato, tal mudança impacta nas leituras da realidade e, sobretudo, nela própria.

Segundo Souza, Silva e Silva (2013), os DSS abarcam aspectos muito imediatos da relação sujeito/sociedade, ainda evidenciando pouco sobre as bases da ordem societária e, sobretudo, sobre a dinâmica capital-trabalho. O termo vulnerabilidade, por si só, é controverso. A vulnerabilidade social, enquanto categoria, emerge, em meados de 1990 pautada por organismos como o Banco Mundial (BM) - dado o esgotamento da "pobreza" enquanto categoria estritamente econômica, que visava a orientação de políticas sociais. Ainda com a mudança de categoria, o foco principal da compreensão continuou sendo o de mapeamento dos desprovidos ao invés de uma compreensão dos determinantes dessa pobreza. Logo, uma definição de vulnerabilidade a partir de seu viés econômico e puramente descritivo (Monteiro, 2011).

Além disso, os DSS, por vezes, apresentam como proposta de superação às iniquidades em saúde, o desenvolvimento econômico e podem contribuir, de forma ideológica, para a reprodução de elementos da estrutura capitalista desigual. Logo, não abarcam uma categoria de totalidade central - como a classe, nem tampouco rompem com as raízes da exploração ou analisam a determinação central no processo saúde doença, que é a pauperização (compreendida aqui como expressão da questão social) (Souza, Silva, \& Silva, 2013). 
O conceito de pobreza também possui diversas concepções não consensuais. A partir da década de 1970, a concepção predominante foi a de necessidades básicas, abandonando o critério exclusivo de renda e considerando outras necessidades como educação, saneamento e habitação. Tal concepção é um marco, pois foi adotada por instituições como o Fundo Monetário Internacional (FMI) e o BM visando estabelecer resultados e objetivos em termos de qualidade de vida. Houve um esforço entre as décadas de 1970 e 1980 para a criação de um indicador social sintético que avaliasse tais objetivos, havendo consenso somente na década de 1990 como o Índice de Desenvolvimento Humano (IDH), baseado na aritmética de três aspectos: a esperança de vida ao nascer, o nível educacional e o PIB per capita. Porém, o índice ainda é deficitário por não captar as nuances da desigualdade e nem considerar as diferenças socioeconômicas dos diferentes países (Rocha, 2006). Nas políticas públicas de assistência social, o termo vulnerabilidade surge como possibilidade de abarcar as múltiplas determinações, a partir de uma perspectiva de integralidade, no contex to de pessoas com restrito - ou nenhum - acessos a direitos. Logo, visa romper com o caráter estritamente monetário de pobreza. Assim, apesar das imprecisões teóricas, o termo é importante para a compreensão da realidade dinâmica (Carmo \& Guizardi, 2018).

Ugá (2004), ao analisar como a categoria pobreza se fez central nas formulações de políticas sociais do BM, afirma que, a partir de 2001, a pobreza passa a ser considerada um fenômeno multifacetado que considera a vulnerabilidade e a exposição ao risco. Assim, o conceito de vulnerabilidade ainda está intimamente ligado ao de risco, mesmo que sendo diferentes, visto que a vulnerabilidade envolve exposição a riscos e "baixa capacidade" de enfrentamento a tais riscos. A pobreza, nessa leitura, seria a exposição ao risco mais danosa. A aproximação entre as definições dos autores que trabalham com vulnerabilidade a as definições do BM é, porém, é arriscada, visto que este último responde a uma visão neoliberal, que toma o mercado como regulador e que, mais uma vez, individualiza questões macroestruturais e tor na as políticas sociais assistencialistas, compensatórias e focalizadas.

Além disso, a partir de tais concepções, a renda e o consumo, graças à monetarização da economia, continuam sendo, as formas primordiais de mensuração da pobreza, o que evidencia os valores societários desse tempo histórico. Dessa forma, o trabalho com o conceito de pobreza e vulnerabilidade, é normativo, pois implica em dizer sobre o que é aceito ou não em uma sociedade em determinado momento histórico: ou ainda, sobre o que seria uma "vida minimamente digna” (Comim, Porto, Moura, \& Bagolin, 2016, p. 17). Fica evidente, assim, que as concepções hegemônicas sobre pobreza são orientadas por interesses do capital e por cada fase do capitalismo, evidenciando os conflitos de classe.

A pobreza - bem como as trajetórias de vulnerabilidades - surge, então, como "evidência concreta das desigualdades sociais" (Guzzo, 2016, p. 149) e, por isso, deve ser entendida como produto de um determinado tempo histórico e de contingências políticas e econômicas. Exatamente por ser uma condição histórica, não se deve perder de vista seu enfrentamento estrutural, que pressupõe a socialização da riqueza produzida. Para além da necessária leitura que leve em consideração a crítica ao capitalismo, faz-se fundamental compreender que a pobreza gera sofrimentos específicos em uma sociedade de consumo e de valorização da acumulação e tem consequências evidentes, como a população em situação de rua. 


\section{Considerações finais}

O processo de construção da revisão sistemática indicou diferenças significativas nos tipos de estudos, metodologias, número de amostras e, consequentemente, concepções e focos de análise entre as pesquisas estadunidenses e latino-americanas. Enquanto as primeiras tendem a priorizar estudos com grandes amostras com o foco na análise de riscos e na quantificação de dados, onde o conceito de risco pressupõe a culpabilização individual, as pesquisas latinas, por sua vez, tendem a contextualizar as condições de desigualdade socioeconômica, além de buscarem uma resposta para ida às ruas e se utilizam de metodologias participativas, se aproximando mais de uma análise de vulnerabilidades. A categoria raça é sub trabalhada em ambos os casos, aparecendo, em grande parte, como informação estatística. Já a categoria estigma não possui definições claras, sendo muitas vezes utilizada como sinônimo de preconceito e estereótipo, sendo poucos os estudos que trazem um embasamento teórico sobre a temática.

Dessa forma, não se trata somente de uma mudança de terminológica - de risco para vulnerabilidades: trata-se de forma de compreensão e de visão de homem e de sociedade. No que tange a população em situação de rua, os processos de marginalização são mais complexos do que a ausência de lar, e dizem respeito a trajetórias marcadas por opressões e que resultam em quadros de vulnerabilidade. Não é possível, dessa forma, afirmar que, mesmo em não situação de rua, não havia vulnerabilidades outras, como a afetiva, a étnico racial, de gênero, de violências e de discriminação. Todas elas resultam em uma sociabilidade que oprime e se referem à gestão da pobreza na sociedade brasileira.

Logo, compreender tais processos se faz fundamental, porém, como meio, e não somente como fim. Como salientado, a categoria vulnerabilidade, por si, não se sustenta a partir de um debate crítico da sociedade capitalista e do enfrentamento de seus condicionantes. Assim como a categoria se faz diversa, também podem ser diversos os projetos societários que se sustentam a partir dela. A partir de nossa leitura sobre pobreza, no entanto, podemos concluir que a sociedade capitalista, tal como se configura, é, por si só, uma” sociedade de risco", o que pressupõe sua superação. Concluímos, que os estudos precisam avançar na análise dos desdobramentos da organização societária burguesa para saúde - o que inclui compreender os determinantes antes analisados, mas, sobretudo, para a compreensão das bases das desigualdades sociais, a fim de contribuir para uma nova proposta social emancipatória. A compreensão do "estado da arte" da temática, assim, aponta para a necessidade de estudos que considerem tanto as trajetórias de vida e particularidades das pessoas em situação de rua, quanto as vejam como pertencentes e atingidas por condições e valores societários complexos nos âmbitos políticos, sociais e econômicos - portanto, atravessadas pelos marcadores de gênero, raça, sexualidade, classe, entre outros. 


\section{Notas}

1. As porcentagens foram arredondadas para a primeira casa decimal.

2. Há três estudos que não correspondem à divisão entre EUA e América Latina: Inglaterra, França e Gana. Enquanto o primeiro se aproxima das características norte americanas, os dois últimos serão debatidos com os estudos latinos.

3. Os estudos do Canadá foram debatidos nessa categoria.

\section{Referências}

Adorno, R., Rui, T., Silva, S., Malvasi, P., Vasconcellos, M., Ramos, B., \& Godoi, T. (2013). Etnografia da cracolândia: notas sobre uma pesquisa em território urbano. Saúde \& Transformação Social . Health E' Social Change, 4(2), 4-13.

Alcantara, S., Abreu, D., \& Farias, A. (2015). Pessoas em situação de rua: das trajetórias de exclusão social aos processos emancipatórios de formação de consciência, identidade e sentimento de pertença. Revista Colombiana de Psicología, 24(1), 129-143.

Alvarado, R., Guerra, A., \& Mejiai, M. (2013). Las adolescentes de la calle y su percepción de la sociedade. Enfermería Global, 12, 371-380.

Arruza, C. (2015). Considerações sobre gênero: reabrindo o debate sobre patriarcado e/ou capitalismo. Revista Outubro, 23. 33-58. Recuperado de http://outubrorevista.com.br/wp-content/uploads/2015/06/2015 1 04 Cinzia-Arruza.pdf

Ayres, J., França, I., Calazans, G., \& Saletti, H. (2009). O conceito de vulnerabilidade e as práticas em saúde: novas perspectivas e desafios. In D. Czeresnia \& C. Freitas (Coords.), Promoção da Saúde: conceitos, reflexões, tendências (pp. 121- 143). Rio de Janeiro: Fiocruz.

Barata, R., Carneiro, N., Ribeiro, M., \& Silveira, C. (2015). Desigualdade social em saúde na população em situação de rua na cidade de São Paulo. Saúde e Sociedade, 24(Supl.1), 219-232

Bassuk, E., Buckner. J., Weinreb. L., Browne, A., Bassuk, S., Dawson, R., \& Perloff, J. (1997). Homelessness in female-feaded families: childhood and adult risk and protective factors. American Journal of Public Health, 87(2), 241-248.

Bobashev, G., Zule, W., Osilla, K., Kline, T., \& Wechsberg, W. (2009). Transactional sex among men and women in the south at high risk for HIV and other STIs. Journal of Urban Health, 86. Recuperado de https://pdfs.semanticscholar.org/1716/9068992a122f2do89boedocdd9f26 9e6f334.pdf

Brêtas, A. C. P., Marcolan, J. F., Rosa, A. S., Fernandes, F. S. L., \& Raizer, M. V. (2010). Quem mandou ficar velho e morar na rua? Revista da Escola de Enfermagem da USP, 44(2), 476-481.

Carmo, M. \& Guizardi, F. (2018). O conceito de vulnerabilidade e seus sentidos para as políticas públicas de saúde e assistência social. Cadernos de Saúde Pública, 34(3). e00101417, 2018. ISSN 0102-311X.

Chacón, R., Silva, M., Guzmán-Facundo, F., Serrano-Cazorla, R., \& Bencomo, A. (201 1). Significado del consumo de drogas para las adolescentes de la calle, en la ciudad de Valencia, Venezuela. Revista Latino-Americana de Enfermagem, 19(n.spe), 746-752. 
Comim, F. V., Porto, S., Moura, J., \& Bagolin, I. (2016). Sem pobreza, mas com "fomes": visões econômicas e fisiológicas. In V. Ximenes, B. Nepomuceno, E. Cidade, \& J. Moura (Orgs.), Implicações Psicossociais da pobreza: diversidades e resistências (pp. 17-44). Fortaleza: Expressão Gráfica e Editora.

Ferreira, R., Oliveira, M., Kantorski, L., Coimbra, V., \& Jardim, V. (2015). Gift theory among groups of users of crack and other drugs. Texto E Contexto - Enfermagem, 24(2), 467-475.

Fleury-Teixeira, P. \& Bronzo, C. (2010). Determinação social da saúde e política. In R. Nogueira (Org.), Determinação social da saúde e Reforma Sanitária (pp. 37-59). Rio de Janeiro: Cebes.

Galvão, C., Sawada, N., \& Trevizan, M. (2004). Revisão sistemática: recurso que proporciona a incorporação das evidências na prática da enfermagem. Revista Latino Americana de Enfermagem, 12(3), 549-556.

Gattis, M. (2013). An ecological systems comparison between homeless sexual minority youths and homeless heterosexual youths. J Soc Serv Res., 39(1), 38-49.

Giraldo, A., Forero, C., Hurtado, M., Ochoa, J., Suàrez, L., \& Valencia, A. (2008). Un viaje que puede controlarse: consumo de drogas en niños en situación de calle. Revista Facultad Nacional de Salud Pública, 26, 11-17.

Grangeiro, A., Holcman, M., Onaga, E., Alencar, H., Placco, A., \& Teixeira, P. (2012). Prevalência e vulnerabilidade à infecção pelo HIV de moradores de rua em São Paulo, SP. Revista de Saúde Pública, 46(4), 674-684.

Guzzo, R. (2016). A (des)igualdade social e a Psicologia: uma perspectiva para o debate sobre a pobreza. In V. Ximenes, B. Nepomuceno, E. Cidade, \& J. Moura (Orgs.), Implicações Psicossociais da pobreza: diversidades e resistências (pp. 149-162). Fortaleza: Expressão Gráfica e Editora.

Hennessey, K., Bangsberg, D., Weinbaum, C., \& Hahn, J. (2009). Hepatitis A seroprevalence and risk factors among homeless adults in San Francisco: ahould aomelessness be included in the risk-based strategy for vaccination? Public Health Reports, 124(6), 813-817.

Janczura, R. (2012). Risco ou vulnerabilidade social? Textos \& Contextos (Por to Alegre), 11 (2), 301-308.

Kecojevic, A., Wong, C., Schrager, S., Silva. K., Bloom. J., Iverson, E., \& Lankenau S. (2012). Initiation into prescription drug misuse: differences between lesbian, gay, bisexual, transgender (LGBT) and heterosexual high-risk young adults in Los Angeles and New York. Addict Behav, 37(11), 1289-1293.

Kowarick, L. (2003). Sobre a vulnerabilidade socioeconômica e civil: Estados Unidos, França e Brasil. Revista Brasileira de Ciências Sociais, 18(51), 61-86.

Laguardia, J. (2004). O uso da variável «raça” na pesquisa em saúde. PHYSIS: Rev. Saúde Coletiva (Rio de Janeiro), $14(2), 197-234$.

Lankenau. S., Bloom, J., \& Shin, C. (2010). Longitudinal trajectories of ketamine use among young injection drug users. Int J Drug Policy, 21(4), 306-314.

Lewis, M., Leake, B., Giovannoni, J., Rogers, K., \&, Monahan, G. (1995). Drugs, poverty, pregnancy, and foster care in Los Angeles, California, 1989 to 1991. West J Med, 163(5), 430-440.

Martins, R. (2002). Uma tipologia de crianças e adolescentes em situação de rua baseada na análise de aglomerados (Cluster Analysis). Psicologia: Reflexão e Crítica, 15(2), 25 1-260.

Mayora, M. (2016). O crack e a rua. In J. Souza (Org.), Crack e exclusão social (pp. 137-162). Brasília, DF: Ministério da Justiça e Cidadania, Secretaria Nacional de Política sobre Drogas. 
Mizen, P. \& Ofosu-IKusi, Y. (2014). Agência como vulnerabilidade: explicando a ida das crianças para as ruas de Acra. Linhas Críticas, 20(41), 81-101.

Monteiro, S. (2011). O marco conceitual da vulnerabilidade social. Sociedade em Debate (Pelotas), $17(2), 29-40$.

Moura, Y., Silva, E., \& Noto, A. (2009). Redes sociais no contexto de uso de drogas entre crianças e adolescentes em situação de rua. Psicologia em Pesquisa, 3(1), 31-46.

Moura, J., Ximenes, V., \& Sarriera, J. (2014). A construção opressora da pobreza no Brasil e suas consequências no psiquismo. Quaderns de Psicologia, 16(2), 85-93.

Moura, J. F., Ximenes, V. M., \& Sarriera, J. C. (2013). Práticas de discriminação às pessoas em situação de rua: histórias de vergonha, de humilhação e de violência em Fortaleza, Brasil. Revista de Psicología, 22(2) 18-28.

Nogueira, R. (2010). A determinação objetal da doença. In Determinação Social da Saúde e Reforma Sanitária (pp. 135-150). Rio de Janeiro: Cebes.

Nunes, E. \& Andrade, A. (2009). Adolescentes em situação de rua: prostituição, drogas e HIV/ AIDS em Santo André, Brasil. Psicologia E Sociedade, 21(1), 45-54.

Nyamathi, A., Dixon, E., Robbins, W., Smith, C., Wiley, D., Leake, B., Longshore, D., \& Gelberg, L. (2012). Risk factors for hepatitis C virus infection among homeless adults. J Gen Intern Med, $17(2), 134-143$.

Parker, R. \& Aggleton, P. (2001). Estigma, discriminação e Aids. Rio de Janeiro: Lidador.

Riley, E., Moore, K., Sorensen, J., Tulsky, J., Bangsberg, D., \& Neilands, T. (2011). Basic subsistence needs and overall health among human immunodeficiency virus-infected homeless and unstably housed women. American Journal of Epidemiology, 174(5), 515-522.

Rocha, S. (2006). Pobreza no Brasil: afinal, do que se trata? Rio de Janeiro: Editora FGV.

Ronzani, T. \& Furtado, E. (2010). Estigma social sobre o uso de álcool. Jornal Brasileiro de Psiquiatria, 59(4), 326-332.

Rosa, A. \& Brêtas, A. (2015). Violence in the lives of homeless women in the city of São Paulo, Brazil. Interface - Comunicação, Saúde, Educação, 19(53), 275-285

Rui, T. (2006). "Só se vive uma vez": uma reflexão acerca de distintas concepções e práticas do uso de "drogas". Revista Mediações, 11(2), 187-202.

Sánchez, A. \& Bertolozzi, M. (2007). Pode o conceito de vulnerabilidade apoiar a construção do conhecimento em Saúde Coletiva? Ciência Ẽ Saúde Coletiva, 12(2), 319-324.

Sarcinelli, A. (2011). Infancias marginales, los márgenes de la infancia. Trayectorias de muchachos en situación de calle em el noreste brasileño. Alteridades, 21(42), 91-101.

Shoptaw, S., Weiss, R., Munjas, B., Hucks-Ortz, S. D. Y., Sherry Larkins, C., Victorianne, G., \& Gorbach, P. (2009). Homonegativity, substance use, sexual risk behaviors, and HIV status in poor and ethnic men who have sex with men in Los Angeles. Journal of urban health, 86(Supl.1), 77-92.

Souza, D., Silva, S., \& Silva, N. (2013). Determinantes Sociais da Saúde: reflexões a partir das raízes da "questão social”. Saúde e Sociedade, 22(1), 44-56.

Souza, J. \& Kantorski, L. P. (2007). Embasamento político das concepções e práticas referentes às drogas no Brasil. SMAD. Revista eletrônica saúde mental álcool e drogas, 3(2), 1-16. Recuperado de http://pepsic.bvsalud.org/scielo.php?pid=S1806-69762007000200003\&script $=$ sci arttext 
Sunkel, G.(2006). El papel de la familia en la protección social en América Latina. Santiago de Chile: Empreso en Naciones Unidas.

Ugá, V. (2004). A categoria "pobreza" nas formulações de política social do Banco Mundial. Rev. Sociologia Política, 23, 55-62.

Varanda, W. \& Adorno, R. (2004). Descartáveis urbanos: discutindo a complexidade da população de rua e o desafio para políticas de saúde. Saúde e Sociedade, 13(1), 56-69.

Vernaglia, T., Vieira, R., \& Cruz, M. (2015). Usuários de crack em situação de rua - características de gênero. Ciência E̊ Saúde Coletiva, 20(6), 1851-1859.

Vogenthaler, N., Hadley, C., Lewis, S., Rodriguez, A., Metsch, L. \&, Del Rio, C. (2010). Food insufficiency among HIV-infected crack-cocaine users in Atlanta and Miami. Public Health Nutr., 13(9), 1478-1484.

Wechsberg, W., Lam, W., Zule, W. \&, Bobashev, G. (2004). Efficacy of a woman-focused intervention to reduce HIV risk and increase self-sufficiency among African American crack abusers. American Journal of Public Health, 94(7). 1165-1173.

Weiser, S., Dilworth, S., Neilands, T., Cohen, J, Bangsberg, T. \&, Riley, E. (2006). Genderspecific correlates of sex trade among homeless and marginally housed individuals in San Francisco. Journal of urban health, 83(4), 736-740.

Wenzel, S., Hsu, H., Zhou, A. \&, Tucker, J. (2012). Are social network correlates of heavy drinking similar among black homeless youth and white homeless youth? Journal of studies on alcohol and drugs, 73(6), 885-889.

Whittle, H., Palar, K., Napoles, T., Hufstedler, L., Ching, I., Hecht, F., Frongillo, E. et al. (2015). Experiences with food insecurity and risky sex among low-income people living with HIV/ AIDS in a resource-rich setting. Journal of the International AIDS Society, 18. doi: 10.7448 / IAS.18.1.20293.

Wood, D., Valdez, B., Hayashi, T., \& Shen, A. (1990). Homeless and housed families in Los Angeles: a study comparing demographic, economic, and family function characteristics. AJPH, 80, 1049-1053. 


\section{KÍSSILA TEIXEIRA MENDES \\ https://orcid.org/0000-0002-7817-599X}

Doutoranda em Psicologia pela Universidade Federal de Juiz de Fora (UFJF). Mestra em Psicologia pela UFJF. Psicóloga pela Faculdade Machado Sobrinho. Bacharela em Ciências Humanas e em Ciências Sociais pela UFJF.

Endereço: Departamento de Psicologia, Instituto de Ciências Humanas. Campus da Universidade Federal de Juiz de Fora - Rua José Lourenço Kelmer, s/no - São Pedro, Juiz de Fora - MG, 36036-900.

E-mail: mendes.kissilateixeira@gmail.com

\section{TELMO MOTA RONZANI \\ https://orcid.org/O000-0002-8927-5793}

Doutorado em Ciências da Saúde pela Universidade Federal de São Paulo (2005), Pós-doutorado pela Universidade de São Paulo (USP) e estágio pósdoutoral pela University of Connecticut Health Center (UCHC) (2007) na área de álcool e outras drogas. Professor da Universidade Federal de Juiz de Fora, consultor ad hoc da Secretaria Nacional de Políticas Sobre Drogas (SENAD).

E-mail: tm.ronzani@gmail.com

\section{FERNANDO SANTANA DE PAIVA \\ https://orcid.org/0000-0002-6030-9777}

Psicólogo e mestre em Saúde Coletiva pela Universidade Federal de Juiz de Fora (UFJF). Doutor em Psicologia (Área de Concentração em Psicologia Social) pela Universidade Federal de Minas Gerais (UFMG). professor adjunto A do Departamento de Psicologia da Universidade Federal de Juiz de Fora (UFJF).

E-mail: fernandosantana.paiva@yahoo.com.br

\begin{tabular}{|c|c|}
\hline Histórico & $\begin{array}{l}\text { Submissão: 08/09/2016 } \\
1^{\text {a }} \text {. Revisão: 06/02/2019 } \\
2^{\text {a. }} \text {. Revisão: 26/6/2019 } \\
\text { Aceite: 12/11/2019 }\end{array}$ \\
\hline $\begin{array}{l}\text { Contribuição } \\
\text { dos autores }\end{array}$ & $\begin{array}{l}\text { Concepção: KTM, TMR, FSP } \\
\text { Coleta de dados: KTM } \\
\text { Análise de dados: KTM } \\
\text { Elaboração do manuscrito: KTM. TMR, FSP } \\
\text { Final aprovação do manuscrito: KTM, TMR, FSP }\end{array}$ \\
\hline Financiamento & $\begin{array}{l}\text { Telmo Mota Ronzani é bolsista Produtividade CNPq. } \\
\text { Kíssila Teixeira Mendes foi bolsista de demanda social da } \\
\text { CAPES durante toda a execução da pesquisa. } \\
\text { FAPEMIG PPSUS APQ-03256-13 }\end{array}$ \\
\hline $\begin{array}{l}\text { Consentimento de } \\
\text { uso de imagem }\end{array}$ & Não se aplica. \\
\hline
\end{tabular}

